\title{
Conversion Surgery in Gastric Cancer Carcinomatosis
}

\begin{abstract}
Paolo Morgagni ${ }^{1}$, Leonardo Solaini ${ }^{1,2}$, Luca Saragoni ${ }^{3}$, Manlio Monti ${ }^{4 *}$, Martina Valgiusti ${ }^{4}$, Giovanni Vittimberga ${ }^{1}$, Giovanni Luca Frassineti ${ }^{4}$, Massimo Framarini ${ }^{1}$ and Giorgio Ercolani ${ }^{1,2}$

${ }^{1}$ General and Oncologic Surgery, "Morgagni-Pierantoni" Hospital, Forli, Italy, ${ }^{2}$ Department of Medical and Surgical Sciences, University of Bologna, Bologna, Italy, ${ }^{3}$ Pathology Unit, "Morgagni-Pierantoni" Hospital, Forli, Italy, ${ }^{4}$ Department of Medical

Oncology, IRCCS Istituto Romagnolo per lo Studio dei Tumori (IRST) "DinoAmadori", Meldola, Italy
\end{abstract}

Background: After the REGATTA trial, patients with stage IV gastric cancer could only benefit from chemotherapy $(\mathrm{CHT})$. However, some of these patients may respond extraordinarily to palliative chemotherapy, converting their disease to a radically operable stage. We present a single centre experience in treating peritoneal carcinomatosis from gastric cancer.

\section{OPEN ACCESS}

Edited by:

Luigi Marano,

University of Siena, Italy

Reviewed by:

Fausto Rosa,

Catholic University of the Sacred

Heart, Rome, Italy

Chukwuemeka Ihemelandu, MedStar Washington Hospital Center,

United States

*Correspondence:

Manlio Mont

manlio.monti@irst.emr.it

Specialty section:

This article was submitted to Gastrointestinal Cancers: Gastric and

Esophageal Cancers,

a section of the journal

Frontiers in Oncology

Received: 11 January 2022

Accepted: 08 February 2022

Published: 08 March 2022

Citation:

Morgagni P, Solaini L, Saragoni L, Monti $M$, Valgiusti $M$, Vittimberga $G$,

Frassineti GL, Framarini $M$ and

Ercolani G (2022) Conversion Surgery

in Gastric Cancer Carcinomatosis.

Front. Oncol. 12:852559.

doi: 10.3389/fonc.2022.852559
Methods: All patients with stage IV gastric cancer with peritoneal metastases as a single metastatic site operated at a single centre between 2005 and 2020 were included. Cases were grouped according to the treatment received.

Results: A total of 118 patients were considered, 46 were submitted to palliative gastrectomy (11 were considered M1 because of an unsuspected positive peritoneal cytology), and 20 were submitted to Hyperthermic Intraperitoneal Chemotherapy (HIPEC) because of a $<6$ Peritoneal Cancer Index (PCI). The median overall survival (OS) after surgery plus HIPEC was 46.7 (95\% Cl 15.8-64.0). Surgery (without HIPEC) after CHT presented a median OS 14.4 (8.2-26.8) and after upfront surgery 14.7 (10.9-21.1). Patients treated with upfront surgery and considered M1 only because of a positive cytology, had a median OS of 29.2 (25.2-29.2). The OS of patients treated with surgery plus HIPEC were 60.4 months (9.2-60.4) in completely regressed cancer after chemotherapy and $31.2(15.8-64.0)$ in those partially regressed $(p=0.742)$.

Conclusions: Conversion surgery for peritoneal carcinomatosis from gastric cancer was associated with long survival and it should always be taken into consideration in this group of patients.

Keywords: gastric cancer, conversion surgery, HIPEC, positive peritoneal cytology, metastatic disease

\section{INTRODUCTION}

Peritoneal carcinomatosis is the most frequent metastatic site in gastric cancer (1). The findings from the REGATTA trial (2) indicate that patients with stage IV gastric cancer could only benefit from chemotherapy, regardless of the metastatic site; however, in other studies, these patients may respond extraordinarily to palliative chemotherapy, converting their disease to a radically operable stage (3) and showing promising results in a much selected group (3-8). 
Considering the peritoneal metastatic site, a radical procedure associated with Hyperthermic Intraperitoneal Chemotherapy (HIPEC) seemed to be a valuable option to improve survival (6). However, the Japanese PHOENIX trial, which reported results comparing intraperitoneal/intravenous versus intravenous preoperative treatment failed to find significant differences between these procedures (9).

Interestingly, there is a different approach to HIPEC or conversion surgery by Eastern and Western authors; whereas Eastern authors presented studies where gastrectomy was proposed after chemotherapy and only if a second laparoscopy could confirm the absence of carcinomatosis, the Western ones considered HIPEC in cases which presented a minimal carcinomatosis [peritoneal cancer index (PCI) <6)] after palliative chemotherapy (10).

In this context, this study aims to present an Italian single center experience on patients with peritoneal carcinomatosis as the single metastatic site.

\section{MATERIAL AND METHODS}

\section{Patient Recruitment}

Between 2005 and 2020, 913 patients were operated on for gastric cancer at the "Morgagni-Pierantoni" General Hospital, in Forlì. Of these, 118 presented peritoneal metastases as a single metastatic site. These cases were all regularly discussed at multidisciplinary meetings during which different approaches were explored from the upfront surgery to conversion according to the guidelines in force at that time

These 118 patients were grouped according to the treatment received: a) patients submitted to upfront surgery and b) patients submitted to palliative chemotherapy and then surgically reevaluated for resection alone or resection plus HIPEC (11). The peritoneal status was measured with the PCI. Patients with nonperitoneal distant metastases were excluded. Morbidity was classified in accordance with the Clavien-Dindo classification (12).
All surgically treated patients were then submitted to postoperative chemotherapy. Tumor stage was presented according to the Union for International Cancer Control (UICC)/ American Joint Committee on Cancer (AJCC) (13).

All procedures performed in this study were in accordance with the ethical standard of the Area Vasta Romagna Ethics Committee (approval n ${ }^{\circ} 5707 / 2020$-I.5/264 on July 3, 2020), with the 1964 Declaration of Helsinki and its later amendments, and with the Good Clinical Practice (GCP) guidelines. Informed consent from patients was collected as instructed.

\section{Statistical Analysis}

Continuous data was presented as the median and interquartile range (IQR). Fisher's exact test was used to compare categorical variables which were presented as numbers and percentages. The Kaplan-Meier curve was used to calculate survival rates, and differences in survival rates between subgroups were assessed by the log-rank test. Overall survival was defined as the time between surgery and death or last follow-up. The median follow-up and IQR was found using the Kaplan-Meier function as suggested by Schemper and Smith (14). A 95\% confidence interval (CI) was reported when required. Analyses were performed with MedCalc ${ }^{\circledR}$ for Windows ${ }^{\circledR}$ (version 10.2.0.0; MedCalc Software, Ostend, Belgium).

\section{RESULTS}

In total, 118 patients with peritoneal carcinomatosis as the exclusive metastatic site were included in the study. The type of treatment received is shown in the flow-chart, Figure 1. Clinicopathological characteristics and operative details are presented in Table $\mathbf{1 .}$

Of the 79 patients treated with upfront surgery, 33 received only an exploratory laparoscopy because of diffuse carcinomatosis, and 46 had palliative gastrectomy. Eleven of these upfront surgery patients were considered M1 only because of a positive peritoneal cytology without macroscopic carcinomatosis (Figure 1).

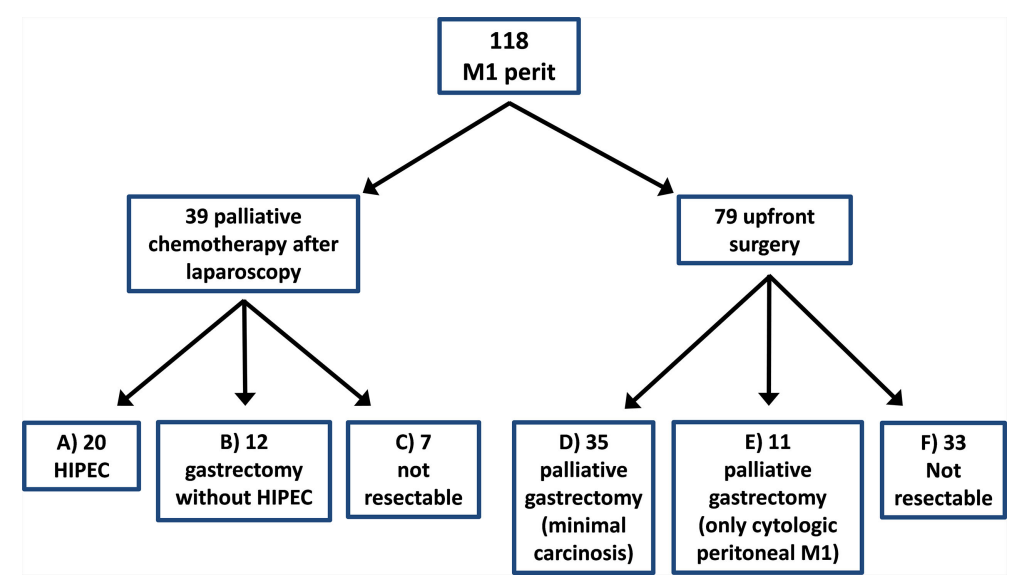

FIGURE 1 | Flow-chart shows the types of treatment. 
TABLE 1 | Patients' characteristics.

\begin{tabular}{|c|c|c|c|c|}
\hline Variables & $\begin{array}{l}\text { Palliative CT+ HIPEC } \\
(\text { No. }=20)\end{array}$ & $\begin{array}{l}\text { CT+ surgery } \\
\text { No. }=12\end{array}$ & $\begin{array}{l}\text { Upfront surgery } \\
\text { No. }=46\end{array}$ & $\begin{array}{c}\text { Explorative laparotomy } \\
\text { No. }=40\end{array}$ \\
\hline \multicolumn{5}{|l|}{ Gender } \\
\hline Male & 13 & 8 & 34 & 22 \\
\hline Age & 61.05 (range 29-78) & 66.5 (range 53-77) & 76.7 (range 61-90) & 67.7 (range 37-89) \\
\hline \multicolumn{5}{|l|}{ T stage } \\
\hline cT2 & 0 & 1 & - & - \\
\hline cT4b & 3 & 1 & - & - \\
\hline уто & 3 & 1 & 0 & 0 \\
\hline y†1 & 3 & 0 & 0 & 0 \\
\hline уТ2 & 1 & 0 & 0 & 0 \\
\hline уТЗ & 4 & 4 & 10 & 0 \\
\hline y†4a & 9 & 6 & 32 & 13 \\
\hline$N x$ & 9 & 3 & - & - \\
\hline yNO & 8 & 3 & 1 & - \\
\hline yN1 & 4 & 3 & 6 & - \\
\hline yN2 & 3 & 1 & 8 & - \\
\hline yN3a & 2 & 3 & 14 & - \\
\hline yN3b & 3 & 2 & 17 & - \\
\hline \multicolumn{5}{|l|}{ Lauren histotype } \\
\hline Intestinal & 14 & 5 & 26 & 20 \\
\hline Diffuse & 3 & 6 & 15 & 20 \\
\hline Mixed & 3 & 1 & 5 & \\
\hline \multicolumn{5}{|l|}{ Site } \\
\hline cardias & 5 & - & 9 & 7 \\
\hline fundus & 3 & 1 & 6 & 4 \\
\hline Subtotal & 5 & 4 & 20 & 0 \\
\hline Total & 15 & 8 & 26 & 0 \\
\hline Explorative & 0 & 0 & 0 & 40 \\
\hline \multicolumn{5}{|l|}{ Cytology } \\
\hline Pretreatment pos & 20 & 2 & - & - \\
\hline Pretreatment neg & 0 & 4 & - & - \\
\hline Pretreatment Not det & 0 & 6 & - & - \\
\hline Surgery pos & 10 & 4 & 16 & 18 \\
\hline Surgery neg & 10 & 5 & 4 & 2 \\
\hline Surgery not det & 0 & 3 & 26 & 20 \\
\hline
\end{tabular}

Thirty-nine patients were submitted to an explorative approach and then proposed for oncologic treatment before re-evaluation. Induction chemotherapy included FOLFOX (5-fluorouracile, leucovorin ed oxaliplatin) in 12 cases (30.8\%), PELF (Cisplatin, Epirubicin, Leukovorin, 5-Fluoruracil) in 12 (30.8\%), FLOT (Fluorouracil, Leucovorin, Oxaliplatin and Docetaxel) in 11 (28.2\%) and other different treatments in the remaining 4 cases (10.2\%). At reevaluation, twenty of these were found as partially $(n=10)$ or totally regressed $(n=10)$ and were submitted to surgery plus HIPEC; twelve patients were submitted to palliative surgery and seven could not be operated on because of progression during treatment.

Clavien-Dindo $>2$ complications rate was $20.0(n=4)$ in patients treated with HIPEC and $13(37.1 \%)$ in patients who had upfront surgery $(p=0.234)$. Mortality was similar between the groups ( 1 death after pancreatitis and leakage vs. 2 deaths due to leakage/bleeding and perforation).

Kaplan-Meier curves are shown in Figure 2. Patients who had surgery plus HIPEC after chemotherapy had a median OS of 46.7 


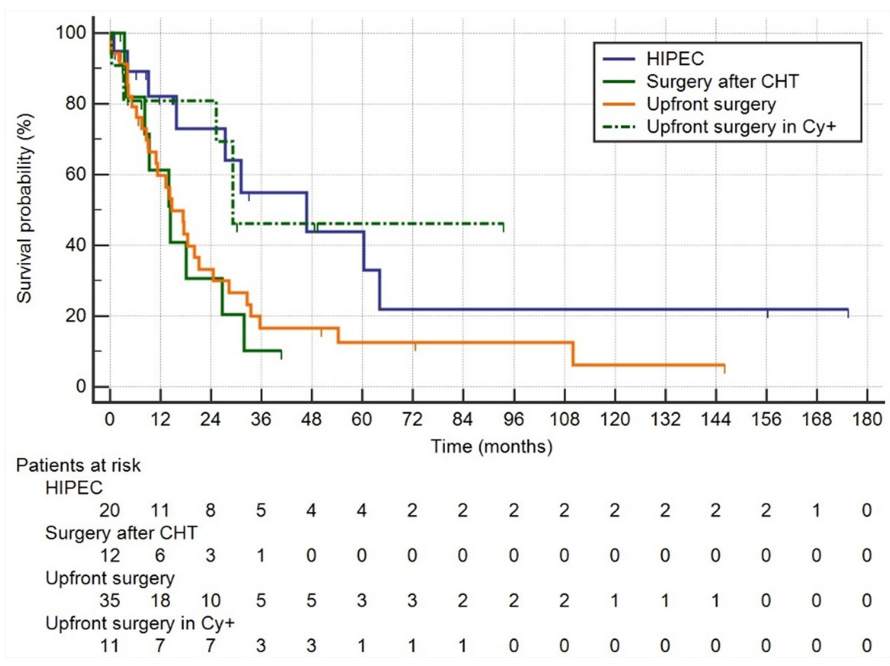

FIGURE 2 | Group1: HIPEC, palliative chemotherapy followed by surgery plus HIPEC; Group 2: surgery after CTH without HIPEC; Group 3: upfront surgery; Group 4: upfront surgery in positive Cy+ only.

months (95\% CI 15.8-64.0), those who had surgery after CHT 14.4 (8.2-26.8), those after upfront surgery 14.7 (10.9-21.1), and finally those who had upfront surgery for positive peritoneal cytology had a median survival of 29.2 months (14.7-29.2) $(p=$ 0.050). Median follow-up was 50 months (IQR 15-110).

Among the 20 patients who had R0 surgery plus HIPEC median survival was $60.4(9.2-60.4)$ months in the group $(n=$ 10) who had surgery plus HIPEC after a complete regression of peritoneal carcinomatosis (and peritoneal cytology) following chemotherapy (CR-HIPEC) versus $31.2(15.8-64.0)$ in the patients $(n=10)$ who had a PCI $<6$ or positive peritoneal cytology after chemotherapy followed by surgery plus HIPEC (PR-HIPEC) $(p=0.742)$ (Figure 3).

\section{DISCUSSION}

The treatment of peritoneal carcinomatosis from gastric cancer represents the most challenging and intriguing treatment frontier and only a multidisciplinary approach may help in achieving the best results.

In 2016, Yoshida et al. classified all metastatic patients into four classes, differentiating macroscopic peritoneal involvement

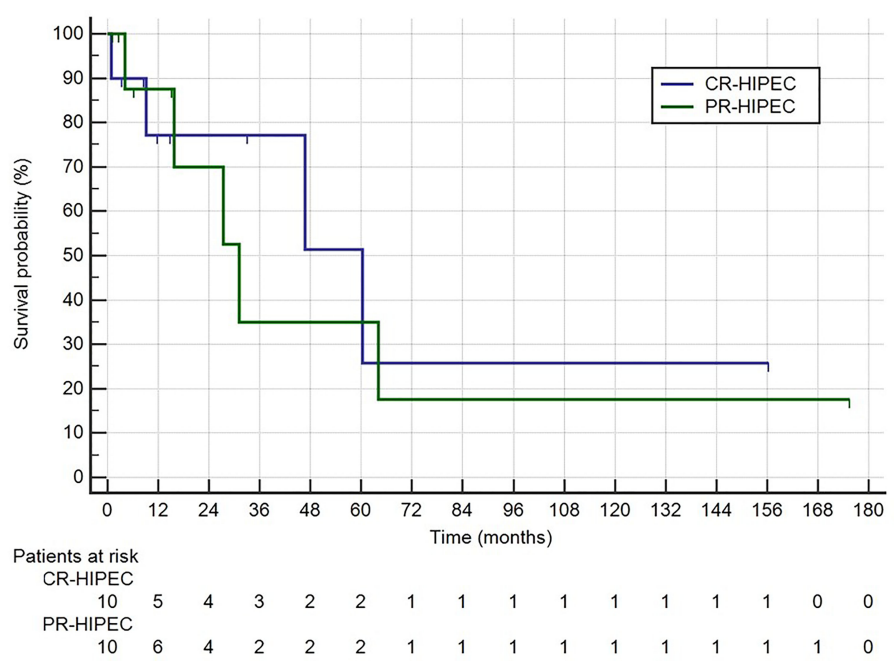

FIGURE 3 | Survival rates for Group 1: CR-HIPEC, completely regressed followed by surgery plus HIPEC; Group 2: PR-HIPEC, partially regressed followed by surgery plus HIPEC. 
or not, and proposing conversion surgery especially for patients without peritoneal involvement (3). The worst results, nonetheless, sometimes gave positive outcomes, as observed by the same author in a selected group of patients with peritoneal involvement with a median survival time of 31.0 for category three (previously unresectable except for local palliation) and 24.7 for category four (previously non curable metastases) (15).

The Korean and Japanese REGATTA trial indicated only chemotherapy for stage IV, and this is currently proposed in Korean guidelines (16).

In Japan some patients can be considered for surgery after chemotherapy and some promising results have been reported with conversion surgery after S1 or intraperitoneal paclitaxel; Yasufuku et al. reported a three-year survival rate of $76.9 \%$ in positive cytology patients (4), and Ishigami et al. reported 30.5 months median survival time after chemotherapy and preoperative HIPEC (5).

Unfortunately, in the West, gastric cancer treatments without the S1 option were associated with the worst results. Peritoneum represents a sort of barrier for chemotherapy and its involvement cannot be approached using conventional treatments. Rau et al., showed a median survival of 18 months in patients treated with surgery plus HIPEC (6) and Passot et al. showed similar results (17).

This poor prognosis is generally due to late diagnosis; early stage carcinomatosis, which could give some hope for cure, is difficult to be diagnosed as staging laparoscopy, which can help in defining the peritoneal involvement, is rarely performed.

Moreover, peritoneal carcinomatosis can be associated with other hidden metastatic sites often not detected at diagnosis and these metastases cannot be cured with a local peritoneal treatment.

Intraperitoneal hyperthermic chemotherapy failed to show clear benefits in patients with massive involvement and its use has also been discussed for gastric cancer at early stages (10).

In the Japanese trial PHOENIX, HIPEC was preoperatively proposed as intraperitoneal weekly chemotherapy through a port associated with intravenous treatment. In the second arm of this trial, patients received only intravenous chemotherapy; results did not find any advantages for preoperative HIPEC.

Of the specific treatments proposed such as HIPEC, bidirectional, and PIPAC, these probably present some results only for a subset of patients; for the other patients there is sometimes only a lesser amount of ascites $(9,18)$.

Our experience collected patients over a long period of time and some of the approaches were changed; in the last ten years, we generally proposed HIPEC for a very selected group of patients with positive cytology or small carcinomatosis detected at laparoscopy and always performed before preoperative treatment.

All the patients were firstly submitted to systemic palliative chemotherapy, generally with FOLFOX treatment and this was usually performed by our oncologist for metastatic treatment. This approach, which postponed surgery for a median of 3 months, improved the selection of those patients with rapidly advancing cancer by treating them only with chemotherapy and avoiding unnecessary surgery.
Responder patients if not R0, but potentially radically resectable with a PCI $<6$, were submitted to surgical treatment with gastrectomy and HIPEC.

The most interesting result of our study was that survival rates after HIPEC did not significantly differ from patients submitted to surgery and completely regressed after palliative preoperative treatment and those patients operated on even if with only a few carcinomatosis, that is: if R0 could be reached, HIPEC was also proposed to patients with small areas of resectable carcinomatosis. These patients presented results as good as those completely regressed after preoperative chemotherapy.

This approach is completely different from the Asian experience which recommended surgery only to patients with complete regression and it also differs from some Western experiences, which presented extended indications for surgery plus HIPEC (10).

Another surprising result was the good survival rates of patients with positive cytology treated without HIPEC, but with upfront surgery. These patients presented promising outcomes with a median survival of 29.2 months (14.7-29.2).

Considering patients M1 only because of peritoneal positive cytology, good survival rates have been presented also by other authors: in 2019, Kim et al. proposed to classify positive cytology in a particular subset with massive lymphatic involvement $(\mathrm{N} 3 \mathrm{~b})$ patients because of unlike other stage IV carcinosis; these patients present similar survival rates (19).

Even if we generally think that patients with advanced carcinomatosis may be better treated with oncologic treatment as proposed by the Korean REGATTA trial, we could, perhaps, select a subset of patients suitable for good surgical results by using a real multimodal approach.

Our study has a few limitations. First, it is a retrospective study and it carries the bias linked to its nature. Second, during the fifteen year-long study interval, several major changes in the management of stage IV gastric cancer have been introduced and this resulted in the heterogeneity of treatment seen in our analysis. As such, staging laparoscopy was not routinely performed as it was after $2013(20,21)$ and this may have had an impact on the type of treatment received. Finally, it must be observed that the upfront surgery group may have included a higher rate of symptomatic cases and/or elderly patients who were not fit for preoperative treatment. Those factors should be taken into account in the interpretation of the survival curves.

\section{CONCLUSION}

Prognosis in peritoneal metastasis is generally poor; however, the good results observed in the HIPEC subset of patients, gives hope that it will be possible to select some patients fit for surgery and stimulate research in this direction.

\section{DATA AVAILABILITY STATEMENT}

The raw data supporting the conclusions of this article will be made available by the authors, without undue reservation. 


\section{ETHICS STATEMENT}

The studies involving human participants were reviewed and approved by the Area Vasta Romagna Ethics Committee (approval $\mathrm{n}^{\circ} 5707 / 2020-\mathrm{I} .5 / 264$ on July 3rd, 2020). The patients/participants provided their written informed consent to participate in this study.

\section{REFERENCES}

1. Marrelli D, De Stefano A, de Manzoni G, Morgagni P, Di Leo A, Roviello F. Prediction of Recurrence After Radical Surgery for Gastric Cancer: A Scoring System Obtained From a Prospective Multicenter Study. Ann Surg (2005) 241:247-55. doi: 10.1097/01.sla.0000152019.14741.97

2. Fujitani K, Yang HK, Mizusawa J, Kim YW, Terashima M, Han SU, et al. REGATTA Study Investigators. Gastrectomy Plus Chemotherapy Versus Chemotherapy Alone for Advanced Gastric Cancer With a Single NonCurable Factor (REGATTA): A Phase 3, Randomized Controlled Trial. Lancet Oncol (2016) 17:309-18. doi: 10.1016/S1470-2045(15)00553-7

3. Yoshida K, Yamaguchi K, Okumura N, Tanahashi T, Kodera Y. Is Conversion Therapy Possible in Stage IV Gastric Cancer: The Proposal of New Biological Categories of Classification. Gastric Cancer (2016) 19:329-38. doi: 10.1007/ s10120-015-0575-Z

4. Yasufuku I, Nunobe S, Ida S, Kumagai K, Ohashi M, Hiki N, et al. Conversion Therapy for Peritoneal Lavage Cytology-Positive Type 4 and Large Type 3 Gastric Cancer Patients Selected as Candidates for R0 Resection by Diagnostic Staging Laparoscopy. Gastric Cancer (2020) 23:319-27. doi: 10.1007/s10120019-00994-0

5. Ishigami H, Yamaguchi H, Yamashita H, Asakage M, Kitayama J. Surgery After Intraperitoneal Chemotherapy for Gastric Cancer With Peritoneal Metastasis or Positive Peritoneal Cytology Findings. Gastric Cancer (2017) 20(Suppl. 1):128-34. doi: 10.1007/s10120-016-0684-3

6. Rau B, Brandl A, Thuss-Patience P, Bergner F, Raue W, Arnold A, et al. The Efficacy of Treatment Options for Patients With Gastric Cancer and Peritoneal Metastasis. Gastric Cancer (2019) 22:1226-37. doi: 10.1007/s10120-019-00969-1

7. Ministrini S, Bencivenga M, Solaini L, Cipollari C, Sofia S, Marino E, et al. Stage IV Gastric Cancer: The Surgical Perspective of the Italian Research Group on Gastric Cancer. Cancers (Basel) (2020) 12:158. doi: 10.3390/ cancers 12010158

8. Solaini L, Ministrini S, Bencivenga M, D’Ignazio A, Marino E, Cipollari C, et al. Conversion Gastrectomy for Stage IV Unresectable Gastric Cancer: A GIRCG Retrospective Cohort Study. Gastric Cancer (2019) 22:1285-93. doi: 10.1007/s10120-019-00968-2

9. Ishigami H, Fujiwara Y, Fukushima R, Nashimoto A, Yabusaki H, Imano M, et al. Phase III Trial Comparing Intraperitoneal and Intravenous Paclitaxel Plus S1 Versus Cisplatin Plus S1 in Patients With Gastric Cancer With Peritoneal Metastasis: Phoenix GC Trial. J Clin Oncol (2018) 36:1922-30. doi: 10.1200/JCO.2018.77.8613

10. Rau B, Brandl A, Piso P, Pelz J, Busch P, Demtröder C, et al. Peritoneum Surface Oncology Group and Members of the StuDoQ|Peritoneum Registry of the German Society for General and Visceral Surgery (DGAV). Peritoneal Metastasis in Gastric Cancer: Results From the German Database. Gastric Cancer (2020) 23:1-22. doi: 10.1007/s10120-019-00978-0

11. Sugarbaker PH. Peritonectomy Procedures. Ann Surg (1995) 221:29-42. doi: 10.1097/00000658-199501000-00004

12. Dindo D, Demartines N, Clavien PA. Classification of Surgical Complications: A New Proposal With Evaluation in a Cohort of 6336 Patients and Results of a Survey. Ann Surg (2004) 240:205-13. doi: 10.1097/01.sla.0000133083.54934.ae

13. Edge S, Byrd D, Compton C. AJCC Cancer Staging Manual, Edn 7. New York: Springer (2010).

\section{AUTHOR CONTRIBUTIONS}

PM designed the study. LSo performed the statistical analysis. PM, LSo, and MM wrote the paper with input from all authors. All authors listed have made a substantial, direct, and intellectual contribution to the work and approved it for publication.

14. Schemper M. Smith TL. A Note on Quantifying Follow-Up in Studies of Failure Time. Control Clin Trials (1996) 17:343-6. doi: 10.1016/0197-2456 (96)00075-x

15. Yamaguchi K, Yoshida K, Tanahashi T, Takahashi T, Matsuhashi N, Tanaka $\mathrm{Y}$, et al. The Long-Term Survival of Stage IV Gastric Cancer Patients With Conversion Therapy. Gastric Cancer (2018) 21:315-23. doi: 10.1007/s10120017-0738-1

16. Guideline Committee of the Korean Gastric Cancer Association (KGCA) and Development Working Group \& Review Panel. Korean Practice Guideline for Gastric Cancer 2018: An Evidence-Based, Multi-Disciplinary Approach. J Gastric Cancer (2019) 19:1-48. doi: 10.5230/jgc.2019.19.e8

17. Passot G, Vaudoyer D, Villeneuve L, Kepenekian V, Beaujard AC, Bakrin N, et al. What Made Hyperthermic Intraperitoneal Chemotherapy an Effective Curative Treatment for Peritoneal Surface Malignancy: A 25-Year Experience With 1,125 Procedures. J Surg Oncol (2016) 113:796-803. doi: 10.1002/ jso. 24248

18. Gockel I, Jansen-Winkeln B, Haase L, Rhode P, Mehdorn M, Niebisch S, et al. Pressurized Intraperitoneal Aerosol Chemotherapy (PIPAC) in Gastric Cancer Patients With Peritoneal Metastasis (PM): Results of a SingleCenter Experience and Register Study. J Gastric Cancer (2018) 18:379-91. doi: 10.5230/jgc.2018.18.e37

19. Kim SH, Lee HJ, Park JH, Choi JH, Park SH, Choe HN, et al. Proposal of a New TNM Classification for Gastric Cancer: Focusing on Pn3b and CytologyPositive (CY1) Disease. J Gastric Cancer (2019) 19:329-43. doi: 10.5230/ jgc.2019.19.e33

20. Solaini L, Bencivenga M, D’ignazio A, Milone M, Marino E, De Pascale S, et al. Which Gastric Cancer Patients Could Benefit From Staging Laparoscopy? A GIRCG Multicenter Cohort Study. Eur J Surg Oncol (2022). doi: 10.1016/ j.ejso.2022.01.020

21. De Manzoni G, Marrelli D, Baiocchi GL, Morgagni P, Saragoni L, Degiuli M, et al. The Italian Research Group for Gastric Cancer (GIRCG) Guidelines for Gastric Cancer Staging and Treatment: The Italian Research Group for Gastric Cancer (GIRCG) Guidelines for Gastric Cancer Staging and Treatment: 2015. Gastric Cancer (2017) 20:20-30. doi: 10.1007/s10120-0160615-3. 2015

Conflict of Interest: The Handling Editor declared a past co-authorship with the authors GE, LS and PM.

Publisher's Note: All claims expressed in this article are solely those of the authors and do not necessarily represent those of their affiliated organizations, or those of the publisher, the editors and the reviewers. Any product that may be evaluated in this article, or claim that may be made by its manufacturer, is not guaranteed or endorsed by the publisher.

Copyright (C) 2022 Morgagni, Solaini, Saragoni, Monti, Valgiusti, Vittimberga, Frassineti, Framarini and Ercolani. This is an open-access article distributed under the terms of the Creative Commons Attribution License (CC BY). The use, distribution or reproduction in other forums is permitted, provided the original author(s) and the copyright owner(s) are credited and that the original publication in this journal is cited, in accordance with accepted academic practice. No use, distribution or reproduction is permitted which does not comply with these terms. 